\title{
Factores condicionantes en la creación informal de nanoempresas: evidencia experimental en Monterrey, México
}

\author{
Conditioning factors in the informal creation of nanoenterprises: \\ Experimental evidence in Monterrey, Mexico
}

\section{Elías Alvarado Lagunas*}

Universidad Autónoma de Nuevo León, México

Recibido el 13 de febrero de 2020; aceptado el 27 de noviembre de 2020

Disponible en Internet el: 3 de diciembre de 2020

\section{Resumen}

El emprendimiento de nanoempresas tiene gran importancia en la economía del área metropolitana de Monterrey, Nuevo León, debido a la elevada cantidad de nanoemprendedores o personas autónomas que buscan día con día un sustento familiar decente. La creación de estas unidades económicas se vuelve un tema relevante para el ámbito académico, político y social. Por esta razón, el objetivo de este artículo es analizar los factores que influyen en la creación informal de nanoempresas o empresas unipersonales. Se implementa un experimento de laboratorio controlado con 385 personas autónomas en el que se aplica una matriz de transición y un modelo binario tipo logit. El experimento consta de tres etapas: primero se realiza un análisis de la situación actual, después se revela información y capacita a las personas sobre las ventajas de convertirse en un nanoemprendedor formal y finalmente se realiza un análisis sobre los factores que motivan dicho cambio. Entre los principales hallazgos se encuentra que los factores que motivan a crear una nanoempresa en la informalidad son el desempleo, empleos mal pagados y mejorar su ingreso familiar. No obstante, en el transcurso del experimento, el $22.12 \%$

\footnotetext{
${ }^{*}$ Autor para correspondencia

Correo electrónico: eliaxalvarado@gmail.com (E. Alvarado Lagunas).

La revisión por pares es responsabilidad de la Universidad Nacional Autónoma de México. 
dijo estar dispuesto a transitar hacia la formalidad si existiera un modelo de intervención directa con apoyos desde la gestión hasta la consolidación de una nanoempresa.

Código JEL: C44, C90, E26, O17

Palabras clave: Nanoempresa; Nanoemprendedor; Economía informal; Matriz de transición; Logit

\begin{abstract}
The entrepreneurship of nanoenterprises is of great importance in the economy of the metropolitan area of Monterrey due to the high number of nano entrepreneurs or self-employed people who are looking for a decent family support every day. The creation of these economic units becomes a relevant issue for the academic, political and social spheres. For this reason, the objective of this article is to analyze the factors that influence the informal creation of nanoenterprises or sole proprietorship. A controlled laboratory experiment with 385 autonomous people is implemented in which a transition matrix and a logit type binary model are applied. The experiment consists of three stages: first an analysis of the current situation is carried out, then information is revealed and people are trained on the advantages of becoming a formal nanoentrepreneur and finally an analysis is carried out on the factors that motivate this change. Among the main findings is that the factors that motivate the creation of a nano business in informality are unemployment, poorly paid jobs and improving their family income. However, in the course of the experiment, $22.12 \%$ are willing to move towards formality if there is a direct intervention model with support from management to the consolidation of a nanoenterprise.
\end{abstract}

JEL Code: C44, C90, E26, $\mathrm{O} 17$

Keywords: Nanoenterprise; Nanoentrepreneur; Transition matrix; Logit

\title{
Introducción
}

La economía informal es un fenómeno común e importante en la actualidad y, si bien se sabe que la informalidad es resultado de empresas y trabajadores que operan fuera de los marcos legales y que traen consigo un bajo crecimiento económico para un país, hay que reconocer que ha sido un "salvavidas" para muchas personas que durante épocas de crisis y ante la falta de empleo se ven motivadas a emprender o autoemplearse dentro del sector informal, donde muchas veces se obtienen más y mejores remuneraciones económicas (Chávez, 2019).

La Organización Internacional del Trabajo (OIT, 2014) señala que cerca del 60\% de la población económicamente activa (PEA) en México no cuenta con seguridad social, lo cual significa que más de la mitad de las personas ocupadas pertenece a la informalidad. Robles y Martínez (2018) sostienen que la informalidad es síntoma de baja productividad y de poco desarrollo en las regiones de la república mexicana, y es común que esté asociada con el 
desempleo, el ambulantaje, la falta de tecnología y la carencia de seguridad social, así como con los efectos negativos en la recaudación fiscal.

En este contexto, la ciudad de Monterrey -en México- no es ajena a este escenario; los nanoemprendedores ${ }^{1}$-entendiéndose con este término a aquellas personas físicas no asalariadas que ejercen en nombre propio una actividad empresarial, profesional o económica sin estar sujetas a un contrato de trabajo- emprenden en la informalidad como una alternativa para generar un mayor ingreso familiar o por la falta de empleo, ya que según datos de la Confederación Patronal de la República Mexicana (COPARMEX) (2019), la tasa de informalidad laboral en Monterrey, es decir, personas que no cuentan con seguro social, es del $37.1 \%$. Por este motivo, es posible señalar que la mayoría de los nanoemprendedores que se incorporan a la economía informal no lo hacen por elección, sino como consecuencia de la falta de oportunidades en la economía formal y por no contar con medios de subsistencia.

Pero, ¿qué pasaría si a un grupo de nanoemprendedores se le otorgara toda la información, capacitación y apoyo para pasar de la informalidad a la formalidad? A partir de esta interrogante, el presente estudio pretende analizar el comportamiento de los nanoemprendedores, asociado en específico a dos preguntas: 1) ¿cambiarán de preferencia los nanoemprendedores a lo largo del experimento?, y 2) ¿qué factores motivan el cambio de preferencias? Para lograr el objetivo del estudio se implementó un experimento de laboratorio controlando el comportamiento de 385 nanoemprendedores que se encuentran en la ciudad de Monterrey en Nuevo León.

Es conveniente mencionar que este artículo se podría enmarcar junto con aquellos que aplican economía experimental, específicamente la técnica o juego del ultimátum a través del cual se analiza que el comportamiento o decisiones de los jugadores -en nuestro caso los nanoemprendedores- pueden cambiar por motivos diferentes a la racionalidad económica -como justicia, equidad, ética e integridad-(Palacio y Parra, 2012). No obstante, la principal diferencia radica en que este trabajo explora el fenómeno de estudio a través de una matriz de transición y un modelo binario tipo logit. La primera permite estudiar los cambios de preferencias que se pueden dar entre los nanoemprendedores con base a las rondas de experimentos que se darán en un ambiente controlado, mientras que el segundo modelo permite comprender el impacto de los factores que propician el cambio agregado en las preferencias de los nanoemprendedores, ante lo cual se supone que la preferencia hacia el emprendimiento de una nanoempresa formal puede ser modelada mediante una variable de respuesta binaria (véase la sección de Metodología para más detalle).

\footnotetext{
${ }^{1}$ En este artículo con el término nanoemprendedor nos referimos a una persona física, nanoempresaria o empresario unitario que, de manera individual, realiza actos de comercio de manera habitual, constituyendo así su propia nanoempresa o negocio, sin conformar sociedades o asociaciones, ni llenando los requisitos exigidos por la Ley General de Sociedades Mercantiles. La creación o emprendimiento de dicha nanoempresa se entiende, entonces, como aquella unidad económica conformada por una persona física no asalariada que ejerce en nombre propio una actividad empresarial, profesional o económica sin estar sujeta a un contrato de trabajo.
} 
Este artículo es pionero en el estudio de análisis sobre el comportamiento de los nanoemprendedores en el estado de Nuevo León. Para ello, se utilizan los datos de un experimento de laboratorio que se llevó a cabo en salas tipo Gesell, reuniendo a los sujetos de estudio por un periodo de seis meses, mientras que otros estudios sólo se enfocan en recabar datos aplicando una encuesta directa de corte transversal. De ahí el interés por hacer una aportación a la investigación de estos temas con esta metodología, ya que, más allá de los resultados, se plantean hipótesis (la información, capacitación y apoyo que se dio a los nanoemprendedores mientras duró el experimento realmente afectaron sus preferencias de los nanoempresarios para participar en la economía formal) que pueden resultar de interés para otras investigaciones en el ámbito de las ciencias administrativas, pues este tipo de experimentos comúnmente son utilizados en disciplinas como la economía y psicología. Además, con este estudio se pretende sentar un precedente relativo a la importancia de contar con alternativas en métodos estadísticos para el análisis de nanoempresas.

\section{Revisión de literatura}

El estudio del fenómeno del emprendimiento de nanoempresas está cobrando una importancia muy peculiar porque influye en el sector informal y, a pesar de que varios autores (Acs, 2006; De la Garma, 2010) sostengan que las actividades realizadas en la informalidad no impactan en el crecimiento económico de un país, es importante reconocer que dicho sector es una parte relevante tanto para de la producción como para el empleo en cualquier país, a la vez que es tema de interés académico, político, económico y social.

A nivel mundial, el emprendimiento es considerado instrumento de combate a la pobreza y el desempleo (Sigalia y Carney, 2012; Rodríguez y Palavicini, 2013; Pazmiño, Merchán y Jiménez, 2018). No obstante, Walton y López (2005), así como Delgado, Cruz y Lince (2019) sostienen que "muchas veces" los emprendimientos que se llevan a cabo son en la economía informal generan "ciertos" efectos positivos; uno de ellos está relacionado con la posibilidad de obtener ingresos para los sectores más pobres de la población, ya que "la mayoría de los más pobres son autoempleados o trabajadores autónomos en unidades muy pequeñas -que pueden ser denominadas nanoempresas o empresas unipersonales- a menudo en empleos inestables y en establecimientos no registrados". Sin embargo, dada la permanencia en la pobreza de estas personas, la economía informal funciona más como una opción de supervivencia que de superación de la pobreza (BM, 2004 y Fundación $1^{\circ}$ de Mayo, 2011).

Baptista, Karaöz y Mendonça (2014), Morales et al. (2015) y Texis, Ramírez y Aguilar (2016) señalan que las personas que hacen negocios porque no tienen otra opción de trabajo son conocidas como emprendedores de necesidad. Siguiendo este contexto, en el presente estudio se denomina "nanoemprendedores" a las personas que se encuentran en esta condi- 
ción, ya que, según Lejarriaga (2003) las nanoempresas son aquellas unidades empresariales constituidas por una sola persona (unipersonal). En este mismo sentido, García y Fernández (2005) y Raydán (2010) agregan que un nanoemprendedor pueden ser catalogados como un empresario individual, persona física o jurídica que profesionalmente y en nombre propio ejerce una actividad de compra-venta de bienes o servicios para un mercado o sector específico. Mientras que Valdés (2004) reconoce al nanoemprendedor como un trabajador autónomo que realiza actividades comerciales por cuenta propia y normalmente no depende de un jefe inmediato, ni tampoco cuenta con una remuneración salarial fija.

El Observatorio Europeo de las PYME (2018) y el Ministerio de Economía y Empresa de España (2002) reconocen que los empresarios individuales o nanoempresarios son personas que dirigen su propio negocio, no tienen salario, viven de los beneficios de sus empresas y tienen plena autonomía de dirección. En otras palabras, los nanoempresarios desarrollan una actividad económica (producción y distribución de bienes y servicios) por cuenta propia, decidiendo de manera individual cómo se ordenan y utilizan los factores de producción, medios, equipo, materia prima e insumos para obtener un fin lucrativo.

Existe una gran diversidad de estudios que tratan de explicar el tema del emprendimiento desde perspectivas sociales y empresariales. En el presente trabajo nos enfocamos en el ámbito empresarial, particularmente en la actividad emprendedora de quienes crearon una nanoempresa. La base teórica sobre el fenómeno de la creación de empresas por la actividad emprendedora está vinculada con los trabajos clásicos de Audretsch y Fristsch (1994) y Keeble y Walker (1994), quienes consideran que la definición y la medición del emprendimiento empresarial pueden ser abordadas desde un enfoque social y personal.

El primer enfoque de ellos se refiere a aquellos factores del entorno que pueden influir directa e indirectamente en la actividad empresarial, tal es el caso de la cultura empresarial de la sociedad en la que se desarrolle el individuo, la estructura sectorial de la actividad económica, el desempleo, la coyuntura económica, el entorno financiero y el capital social, entre otros. Mientras, los factores personales se refieren al entorno familiar, la insatisfacción con el trabajo asalariado, la oportunidad y la capacidad de financiación, la necesidad de conseguir logros, la edad, la formación profesional y la seguridad personal, entre otros. Algunos estudios sobre la actividad emprendedora, la influencia, la motivación y la oportunidad de negocio se han enfocado principalmente en identificar aquellos aspectos o razones que los emprendedores consideran relevantes para crear una empresa. A continuación se describen algunos estudios que nos permiten identificar los elementos que debe cumplir un emprendedor para crear una nanoempresa o negocio.

Gibb y Ritchie (1982) fueron los primeros en establecer los factores determinantes que necesita un emprendedor para crear un nano o micronegocio. Estos autores argumentan que existen cuatro factores claves para obtener un comienzo exitoso: la motivación, la idea o 
mercado, los recursos y las habilidades. Asimismo, Alonso y Galve (2008), Álvarez y Valencia (2008), García, Martínez y Fernández (2010) y Kantis (2003) coinciden con Gibb y Ritchie señalando que toda iniciativa emprendedora en la creación de una empresa depende de la determinación o motivación que tenga la persona, así como también de la observación y oportunidad que aproveche de su entorno.

No hay que olvidar que de los elementos anteriores existen algunos que no son aplicables para el caso de los nanoemprendedores, tales como: la evaluación de la idea y la ejecución de estudios de mercados. Davidsson y Honing (2003) y Kim, Aldrich y Keister (2006) mencionan que la creación de un negocio o empresa no se lleva a cabo de la "noche a la mañana", sino que tiende a ser un proceso de meses, e incluso años, en el cual a las personas que están en dicho proceso se les conoce como emprendedores nacientes quienes pueden tomar la decisión de participar en un mercado formal e informal en el transcurso de su implementación.

Durante el proceso de llevar a cabo el negocio los nanoemprendedores pueden combinar varios factores para su creación. En resumen, estos factores pueden clasificarse en cinco grupos: capital humano, capital social, capital financiero, capital cultural y capital simbólico (Casas e Ibarra, 2013; Nava, 2013). Estos capitales no sólo favorecen la creación de negocios y empresas, sino que permiten la competitividad y pueden ser de tipo tangible e intangible (Fuentes, Osorio y Mungaray, 2016). Por ejemplo, el capital humano se compone de conocimientos y habilidades adquiridas por la trayectoria de la persona y se puede generar vía la educación recibida y aplicarse tanto formal como informalmente (Baptista et al., 2014). Para el primer caso, Davidsson y Honing (2003) y Backes y Moog (2013) argumentan que las personas que posean un grado de escolaridad mayor identificarán de mejor manera las oportunidades empresariales.

Sin embargo, la mayoría de los nanoemprendedores, generalmente, no cuentan con un nivel de escolaridad alto, por lo que la experiencia personal y de trabajos anteriores les permite acumular habilidades y conocimientos para crear un oficio o negocio.

Por su parte, Coleman (1988) y Narayan y Pritchett (1999) destacan que el capital social juega un rol fundamental en la creación de una empresa, ya que es el activo que las personas obtienen al estar en contacto con otros. Es decir, este capital está conformado por la motivación, influencia o enlaces que se obtienen de los compañeros de trabajo, escuela, familia, amigos, etcétera. En lo que se refiere al capital financiero, según Scott (2009), Elston y Audretsch (2011) y Rodríguez (2006), es otro factor significativo para crear un negocio o empresa, y se refiere básicamente a los recursos monetarios que posean o a que tengan acceso las personas. Sin embargo, poseer los recursos financieros no será suficiente para emprender, sino se tiene la mentalidad de empresario. Con este hecho se vincula el enfoque de capital cultural, el cual consiste en la manera de pensar, sentir y de hacer de las personas. Kim et al. (2006), Gelderen, Bosma y Thurik (2001), Quintana (2001) y Pinillos (2001) asocian este 
enfoque con la operacionalización de variables como la edad, educación, estado civil, entorno geográfico y la experiencia laboral y personal, lo cual coincide con los elementos del capital humano y social. Por ejemplo, la influencia de una ciudad como Monterrey en la que existen grandes empresarios y negocios de todo tipo puede impactar en la aspiración de emprender en una persona por emprender, y esta influencia no necesariamente provendría de la familia.

Por último, el capital simbólico representa la influencia o poder que tiene una persona sobre otras, así como el prestigio, la reputación y el estatus social. Anthopoulou (2010) y Scott (2009) sostienen que todas las personas buscan y anhelan dichos valores, los cuales se traducen, en este trabajo, como el elemento motivacional de los nanoemprendedores, ya que, sin duda, este factor permite la separación de las personas que crean nanoempresas de las que no lo hacen. Por otro lado, Kantis, Ishida y Komori (2002) establecen que no todas las personas emprendedoras anhelan estos valores, ya que, al momento de crear una empresa, existen quienes persiguen fines lucrativos y quienes no.

Todo lo anterior permite comprender que la creación de una nanoempresa se puede dar de diferentes maneras porque depende de la combinación de capitales, comportamiento, necesidad y motivación de las personas, cuyo determinante es la intencionalidad de comenzar. A pesar de que en México legal o jurídicamente aún se prohíben las nanoempresas -o empresas o sociedades unipersonales-, dicha prohibición no es explícita en la Ley General de Sociedades Mercantiles, ya que, siguiendo a Garza (2010) al hacer referencia a cada una de las sociedades reguladas por este ordenamiento, se menciona que son sociedades que se constituyen entre "socios", es decir, son referidas de manera plural. Además, en el artículo 89 de dicha ley, correspondiente al capítulo de las sociedades anónimas, expresamente se señala como primer requisito para su constitución que haya dos socios como mínimo. También, la fracción cuarta del artículo 229 determina como causa para disolver una sociedad mercantil que el número de accionistas llegue a ser inferior al mínimo establecido por esta ley, o que las partes de interés se reúnan en una sola persona. En este sentido, y con base en la revisión de literatura sobre el tema, no se ha encontrado evidencia de trabajos en los que se hayan realizado experimentos de laboratorio relacionados con la creación o con los perfiles de nanoemprendedores. Por esta razón, el presente estudio pretende cubrir el vacío de este tipo de análisis en esta temática.

\section{Metodología}

\section{Diseño experimental}

Con el objetivo de cuantificar el comportamiento de los nanoemprendedores en la ciudad de Monterrey en Nuevo León, se aplica un experimento de laboratorio, así como con la finalidad 
de controlar algunos factores socioeconómicos, ideológicos y de entorno para averiguar si estos difieren entre los nanoemprendedores que operan en la informalidad y los que no. De esta manera, es posible contrastar distintas hipótesis, como la diferencia de comportamientos y preferencias de los nanoemprendedores formales frente a los no formales, específicamente cuando se les otorga información, capacitación y apoyo mientras dura el experimento, o viceversa.

El experimento se implementó de octubre de 2017 a marzo de 2018, estableciendo seis meses de trabajo y observación. Dicho experimento se llevó a cabo en tres etapas. La primera etapa consistió en realizar un análisis descriptivo sobre la muestra de 385 nanoemprendedores; ${ }^{2}$ es decir, en este primer acercamiento se recabó y analizó la información a través de un cuestionario que capta las características generales de las personas que tienen una nanoempresa o empresa unipersonal en la región objeto de estudio. Para ello, se aplicó un muestreo de bola de nieve mediante el cual se llevó a cabo el reclutamiento de los participantes; particularmente, se orientó la muestra a personas mayores de edad de cualquier sexo que se encontraran emprendiendo alguna actividad comercial u oficio por cuenta propia. Para reclutar a estos nanoemprendedores, se realizó trabajo de campo, el cual consistió en un acercamiento a sus lugares y zonas de trabajo, así como a sus redes sociales -ya que a través de éstas ofrecen sus productos y servicios- para invitarlos a colaborar en este proyecto de investigación.

En esta primera etapa se les mencionó a las personas participantes que el experimento tendría una duración de un semestre y que se necesitaba su apoyo con la recomendación de otro compañero, amigo o familiar que estuviera en las mismas condiciones y deseara participar en el proyecto experimental, ya que el objetivo era estudiar su comportamiento a lo largo de dos etapas más. Se especificó que la siguiente sesión sería en las instalaciones (de renta) de la consultoría Roheisen, la cual cuenta con salas tipo Gesell, y que tendría una duración aproximada de hasta dos horas en el mes de diciembre. Asimismo, se informó que se les daría una remuneración de 100 pesos por cada sesión, además de obsequiarles un jersey y souvenirs de la Universidad Autónoma de Nuevo León.

En la segunda etapa del estudio se pretendió recoger la preferencia de los nanoempresarios en el emprendimiento individual o autónomo, sobre las posibles ventajas o externalidades positivas de cambiar su estado informal a formal. En particular, esta fase -denominada manipulación experimental- se centró en proporcionar información y capacitación a los nanoemprendedores sobre la transición, los procesos y las ventajas de convertirse en un nanoempresario formal. En esta etapa, los nanoemprendedores fueron conducidos a las salas Gesell donde se explicó a detalle el contenido de la sesión. Se expusieron ejemplos, casos de estudio y de vida real sobre microempresarios que emprendieron en la informalidad, pero que con el tiempo deci-

\footnotetext{
${ }^{2}$ A pesar de que no existe un registro formal de nanoemprendedores o nanoempresarios en la ciudad de Monterrey, en este estudio se hizo el cálculo del tamaño de muestra para una población infinita y para poder establecer una lista de participantes donde la proporción esperada de éxito y de fracaso fuera la misma ( $\mathrm{p}=0.5$ y q=0.5), teniendo en cuenta un nivel de confianza igual a $95 \%$ y un error muestral de 0.05 .
} 
dieron transitar hacia la formalidad y en la actualidad estaban siendo exitosos. Dicha sesión de motivación, explicación y capacitación duró aproximadamente una hora y media y a través de ella se aseguró que los nanoemprendedores conocieran el conjunto de estrategias, procesos y soluciones posibles para transitar hacia la formalidad. Una vez que se expuso lo anterior, se llevó a cabo una sesión de preguntas y respuestas para garantizar que los sujetos de estudio comprendieran la importancia del tema en cuestión. Posteriormente, se aplicó una encuesta sobre la percepción de los nanoemprendedores y se verificó si hubo un cambio de preferencias y en la intención de querer transitar a su nanoempresa hacia la formalidad.

En la tercera y última etapa, se aplicó un cuestionario en línea con la finalidad de recolectar y corroborar la información sociodemográfica y otras características de las personas participantes, tales como las razones por las que conservarían o cambiarían el estado de su nanoempresa, su financiamiento, los principales problemas que enfrentan, ganancias, gastos y sus aspiraciones sobre el futuro. Esta etapa se llevó a cabo con la finalidad de realizar un análisis inferencial sobre los factores que motivan el cambio de preferencias de los nanoemprendedores.

Cabe aclarar que este es un análisis inicial para comprobar si se cumplen algunas de las propuestas planteadas en un estudio de experimento de laboratorio, señalado al inicio. Por lo que, con los resultados del presente experimento y la experiencia adquirida se podrá plantear en el futuro un refinamiento del instrumento y realizar un estudio con una muestra probabilística, para poder generalizar los resultados.

\section{Especificación de los métodos empíricos}

Los datos presentados en este artículo fueron analizados a través de una matriz de transición (MT) y un modelo de elección discreto binario tipo logit (logit). El primero de ellos permite identificar la cantidad de observaciones que, dado el valor específico en una variable aleatoria $X$, "transitan" hacia un valor de la variable aleatoria $Y$ (Budnick, 2007). En otras palabras, en una matriz de transición se indican las probabilidades condicionales de que ocurra un valor específico en la variable $X$ dado un valor específico de la variable $Y$.

Tomando en cuenta lo anterior, si se asume que existen diferentes categorías o formas en las que puede estar una nanoempresa $(D)$ a través de las cuales los nanoemprendedores solamente pueden elegir una, entonces se puede definir una $\mathrm{MT}^{3}$ como: $P=\left[p_{i j}\right]$, la cual indica una matriz de probabilidades que muestra la probabilidad de que un grupo de nanoemprendedores se mantenga en la misma forma o categoría a su nanoempresa (en este caso, $i$ $=j$ ) o que se mueva a una de las otras $D-1$ formas existentes durante un periodo de tiempo

\footnotetext{
${ }^{3}$ Kolman y Hill (2006) señala que la MT debe presentar las siguientes características: a) Todos los elementos de la matriz son no negativos y b) la suma de los elementos de cada fila es igual a uno. Es decir,
} 
dado. De esta manera, cada elemento de la matriz, $p_{i j}$, muestra la probabilidad de que los nanoemprendedores que están en la informalidad $i$ en el periodo $t-1$ puedan cambiar a la formalidad $j$ en el periodo $t$ :

$$
P=\left[\begin{array}{cccc}
p_{11} & p_{12} & \cdots & p_{1 D} \\
p_{21} & p_{22} & \cdots & p_{2 D} \\
\vdots & \vdots & \ddots & \vdots \\
p_{D 1} & p_{D 2} & \cdots & p_{D D}
\end{array}\right]
$$

De la ecuación anterior se puede resaltar que cada celda representa la probabilidad que de un nanoemprendedor transite hacia otra preferencia o forma de crear su nanoempresa, es decir, la MT muestra las probabilidades de transición de lo informal a lo formal, o viceversa, ya que permite construir la transición con $i$ filas (preferencia al inicio del período) y $j$ columnas (preferencia al final del período).

La diagonal de la matriz representa el porcentaje de nanoemprendedores que retuvieron su preferencia desde el periodo inicial hasta el final del experimento, mientras que la intersección de estas celdas representa el porcentaje de los nanoemprendedores que se mantuvieron, aumentaron o disminuyeron en cada ronda. De ahí que las celdas que se encuentran por debajo y encima de la diagonal indican los movimientos en preferencias de una opción de creación de una nanoempresa a otra, lo cual nos indica el cambio de lo informal a lo formal, o viceversa.

Por su parte, el modelo logit permite conocer el impacto que tienen diferentes factores en la intención o cambio de preferencia de los nanoemprendedores en la ciudad de Monterrey, ya que este tipo de modelo nos sirve para cuantificar el comportamiento de variable de respuesta binaria. Martínez (2008) y Pérez (2005) sostienen que para el caso de este tipo de modelo, la función utilizada es logística, por lo que la especificación econométrica es la siguiente:

$$
Y_{i}=\frac{1}{1+e^{-a-b_{k} X_{k i}}}+e_{i}=\frac{e^{a+b_{k} X_{k i}}}{1+e^{a+b_{k} X_{k i}}}+e_{i}
$$

Donde: $Y_{i}$ es una variable de respuesta binaria para $i=1,2, \ldots, N$ observaciones, $X k i$ es el vector de $K$ variables independientes para la i-ésima observación y $b k$ es el vector de $K$ parámetros. A partir de la ecuación (2) se construyen tres modelos logit con el fin de examinar el impacto de diferentes factores en la probabilidad de preferir por una categoría (o forma) de nanoempresa en particular. El primer modelo estima el impacto de las variables independientes para la preferencia de seguir en la informalidad, el segundo para pasar a la formalidad y el tercero mide la indiferencia. Recordemos que en cada modelo la variable dependiente es binaria, con valor de 1 si prefiere la categoría en cuestión, y de 0 si no. 
Para cada modelo se tienen como variables independientes cuatro grupos de variables: 1) aspectos socioeconómicos, agrupados en variables como la edad (muestra la edad del nanoemprendedor en años cumplidos), casado (variable binaria que indica esta condición con el valor de 1), género (variable binaria que identifica si el nanoemprendedor es hombre con el valor de 1), escolaridad (refleja el nivel educativo del nanoemprendedor en una escala de 0 a 9 , donde 0 es no contar con educación y 9 indica el máximo grado de educación, i.e., universidad completa o superior), clase (mide el nivel de ingreso del nanoemprendedor indicando la clase social a la que pertenece en una escala de 1 a 5 , donde 1 es la clase social más alta y 5 la más baja4), inversión (muestra mensualmente el gasto de inversión promedio realizado para la nanoempresa medido en miles de pesos) y ganancias (son las utilidades mensualmente promedio por realizar actividades comerciales o de servicios medidas en miles de pesos); 2) aspectos del entorno, agrupados en variables como las promesas del gobierno (variable binaria con valor de 1 si el nanoemprendedor cambia su preferencia debido a las promesas del gobierno para la formalidad), inseguridad (variable binaria con valor de 1 si el nanoemprendedor cambia su preferencia debido a la inseguridad-extorsión o cobro de piso), desempleo (variable binaria con valor de 1 si el nanoemprendedor inició debido a este factor) y empleos mal pagados (variable binaria con valor de 1 si el nanoemprendedor inició debido a que los empleos que encontró estaban mal pagados); 3) aspectos simbólicos, agrupados en variables como la influencia empresarial (variable binaria con valor de 1 si el nanoemprendedor inició debido a este factor), familiar (variable binaria con valor de 1 si el nanoemprendedor tuvo influencia familiar para iniciar), oportunidad de negocio (variable binaria con valor de 1 si el nanoemprendedor inició por una oportunidad), mejorar ingreso (variable binaria con valor de 1 si el nanoemprendedor inició debido a que quiere mejorar el ingreso familiar) y única forma (variable binaria con valor de 1 si el nanoemprendedor inició debido a que fue la única manera de obtener un ingreso), y 4) preferencias, agrupados con las variables de informalidad, formalidad e indeciso (variables binarias con valor de 1 si el nanoemprendedor prefiere cada uno de los factores, respectivamente).

\section{Resultados}

En esta sección se muestran los resultados descriptivos e inferenciales obtenidos en las tres etapas del experimento con los nanoemprendedores, los cuales nos permitirán conocer cuál era la situación en el momento de contactarlos (primera etapa), así como cuantificar si existe una transición o cambio de preferencias derivado de las presentaciones e interacciones respecto

\footnotetext{
${ }^{4}$ La tipología de clases sociales que se utiliza en este estudio tiene una correspondencia con la propuesta por la AMAI (Asociación Mexicana de Agencias de Investigación de Mercado y Opinión Pública). Para más información, véase https://www.amai.org
} 
a las ventajas de ser un nanoemprendedor formal (segunda etapa) y, por último, analizar los factores que motivaron el cambio de preferencias (tercera etapa) de los nanoemprendedores en la ciudad de Monterrey

\section{Análisis descriptivo aplicado a los nanoemprendedores}

La Tabla 1 muestra los estadísticos descriptivos de frecuencia, donde se aprecia que la mayoría de las personas son hombres $(62.1 \%)$, y que la mayoría de los nano emprendedores encuestados reportó tener entre 30 a 40 años de edad. La mayor parte de ellos argumentó ser soltero (57.9\%), mientras que el resto se encontraba casado. Asimismo, muchos de estos nano emprendedores señalaron que sus ganancias promedio mensual oscilan entre 13 a 19 mil pesos, obteniendo un promedio de la muestra de 15,376.6 pesos. De igual manera, se observan los porcentajes de los participantes según los motivos que los orillaron para ser un nanoempresarios. De manera general, se aprecia que el motivo más frecuente para emprender una nanoempresa fue "el desempleo", "empleos mal pagados", "única forma de ingreso" y "mejorar el ingreso familiar", mientras que el de menor importancia fue "las promesas del gobierno".

Otro resultado interesante que cabe mencionar es el relacionado con los participantes que argumentaron que sus nanoempresas o actividades mercantiles o de servicios no estaban registradas debidamente ante la ley. ${ }^{5}$ Se aprecia que los nanoemprendedores varones $(72.1 \%)$ y solteros (60.6\%) son quienes los que por cuestiones de "tiempos" y "costos" han dejado de acudir ante las instituciones correspondientes para dar de alta de manera legal su nanoempresa, mientras que los nano emprendedores casados (sea hombre o mujer) argumentan estar establecidos formalmente (23.6\%). Por último, aunque no menos importante, el motivo que más lleva a crear y no cumplir con la formalización de la nanoempresa es "el desempleo" y "empleos mal pagados".

\footnotetext{
${ }^{5}$ El $76.4 \%$ de los participantes señala que no se han registrado debidamente porque la constitución formal de un negocio o nanoempresa conlleva una serie de trámites legales y administrativos que son tardados. De ahí, el interés de cambiar este tipo de percepciones con el experimento llevado a cabo en el presente estudio.
} 
Tabla 1

Características generales de los nanoemprendedores en Monterrey

\begin{tabular}{|c|c|c|c|c|c|}
\hline \multicolumn{2}{|r|}{ Componente } & \multicolumn{2}{|c|}{ Total } & \multicolumn{2}{|c|}{ Informalidad } \\
\hline & & Frecuencia & $\%$ & Frecuencia & $\%$ \\
\hline \multirow{3}{*}{ Género } & Hombre & 239 & 62.1 & 212 & 72.1 \\
\hline & Mujer & 146 & 37.9 & 82 & 27.9 \\
\hline & Total & 385 & 100.0 & 294 & 100.0 \\
\hline \multirow{5}{*}{ Edad } & Menos de 21 años & 28 & 7.2 & 17 & 5.8 \\
\hline & De 21 a 29 años & 86 & 22.3 & 71 & 24.1 \\
\hline & De 30 a 40 años & 199 & 51.8 & 154 & 52.4 \\
\hline & Más de 41 años & 72 & 18.7 & 52 & 17.7 \\
\hline & Total & 385 & 100.0 & 294 & 100.0 \\
\hline \multirow[t]{2}{*}{ Estado } & Soltero & 223 & 57.9 & 178 & 60.6 \\
\hline & Casado & 162 & 42.1 & 116 & 39.4 \\
\hline Civil & Total & 385 & 100.0 & 294 & 100.0 \\
\hline \multirow{6}{*}{ Escolaridad } & Sin estudios & 29 & 7.5 & 19 & 6.5 \\
\hline & Primaria & 78 & 20.3 & 68 & 23.1 \\
\hline & Secundaria & 62 & 16.1 & 52 & 17.7 \\
\hline & Preparatoria & 87 & 22.6 & 63 & 21.4 \\
\hline & Estudios universitarios & 129 & 33.5 & 92 & 31.3 \\
\hline & Total & 385 & 100.0 & 294 & 100.0 \\
\hline \multirow{5}{*}{ Ganancias } & Menos de 5 mil pesos & 49 & 12.7 & 35 & 11.9 \\
\hline & De 6 a 12 mil pesos & 116 & 30.2 & 82 & 27.8 \\
\hline & De 13 a 19 mil pesos & 194 & 50.3 & 131 & 44.6 \\
\hline & Más de 20 mil pesos & 26 & 6.8 & 46 & 15.7 \\
\hline & Total & 385 & 100.0 & 294 & 100.0 \\
\hline
\end{tabular}




\begin{tabular}{llrrrr} 
& Mejorar ingreso & 43 & 11.3 & 35 & 11.9 \\
& Promesas & 14 & 3.6 & 9 & 3.1 \\
& Desempleo & 106 & 27.5 & 86 & 29.3 \\
\cline { 2 - 2 } Motivos para ser & Mal pagado & 91 & 23.6 & 71 & 24.1 \\
nanoempresario & Influencia empresarial & 21 & 5.5 & 14 & 4.8 \\
& Influencia familiar & 33 & 8.6 & 27 & 9.1 \\
& Oportunidad de negocio & 29 & 7.5 & 19 & 6.5 \\
& Única forma de ingreso & 48 & 12.4 & 33 & 11.2 \\
& Total & 385 & 100.0 & 294 & 100.0 \\
\hline
\end{tabular}

Fuente: Elaboración propia.

Análisis de los cambios de preferencias en los nanoemprendedores

La Tabla 2 presenta los resultados de la matriz de transición, la cual permite determinar si ha existido un cambio de preferencias por parte de los nanoemprendedores de la primera etapa (situación o perfil actual) a la segunda etapa (experimento aplicado). Antes de interpretar los resultados descrito en la siguiente tabla, es necesario comprender que la muestra de los participantes en este análisis disminuyó a 217 nanoemprendedores, ya que para garantizar que la matriz fuera consistente en las etapas se tuvo que recategorizar las preferencias en cada una de ellas etapas. Es decir, algunos nanoemprendedores entrevistados en la primera etapa no asistieron o no participaron en las etapas posteriores, por lo tanto, se optó por llevar a cabo el análisis con aquellos nanoemprendedores que si fueron entrevistados o participaron en todas las etapas.

En la tabla anterior se puede apreciar que cada celda tiene una combinación de $i$ filas y $j$ columnas, donde a través de las celdas es posible realizar interpretaciones del comportamiento de los nanoemprendedores. Por ejemplo, respecto a los nanoemprendedores que argumentaron estar participando en el mercado laboral o de servicios de manera informal (celda $i=1$ y $j=$ 1), señala una retención (o decisión) de 91 participantes en la segunda etapa, considerando que en la primera etapa a 147 participantes manifestaron su preferencia por esta categoría o manera de laborar (véase la última columna con la etiqueta Total).

Al dividir estos dos números $(91 / 147)$, se observa que la decisión de permanecer en la informalidad es de $61.90 \%$ y representa el $41.93 \%$ del total de los nanoempresarios que decidieron participar en el experimento completo. 
Tabla 2

Matriz de transición de preferencias de los nanoemprendedores

\begin{tabular}{|c|c|c|c|c|}
\hline \multirow[b]{2}{*}{ Preferencia primera etapa } & \multicolumn{3}{|c|}{ Preferencia segunda etapa } & \multirow[b]{2}{*}{ Total } \\
\hline & Informalidad & Formalidad & Indeciso & \\
\hline \multirow{3}{*}{ Informalidad } & 91 & 48 & 8 & 147 \\
\hline & 61.90 & 32.65 & 5.45 & 100.0 \\
\hline & 41.93 & 22.12 & 3.69 & 67.74 \\
\hline \multirow{3}{*}{ Formalidad } & 0 & 47 & 3 & 50 \\
\hline & 0.0 & 94.0 & 6.0 & 100.0 \\
\hline & 0.0 & 21.66 & 1.38 & 23.04 \\
\hline \multirow{3}{*}{ Indeciso } & 0 & 9 & 11 & 20 \\
\hline & 0.0 & 45.0 & 55.0 & 100.0 \\
\hline & 0.0 & 4.15 & 5.07 & 9.22 \\
\hline \multirow{3}{*}{ Total } & 91 & 104 & 22 & 217 \\
\hline & 41.93 & 47.93 & 10.14 & 100.0 \\
\hline & 41.93 & 47.93 & 10.14 & 100.0 \\
\hline
\end{tabular}

Fuente: Elaboración propia.

Para los nanoemprendedores que argumentaron ser formales (celda $i=2, j=2$ ) se observa que de 50 participantes de la primera etapa, 47 mantuvieron su decisión o preferencia, es decir, $94 \%$. Dicha cantidad equivale a $21.66 \%$ del total de entrevistas en las dos etapas. Mientras que la información de la celda $i=3 \mathrm{y} j=3$ muestra a los nanoemprendedores indecisos que se retuvieron en $55 \%$ de los participantes que seleccionaron esta opción en la primera etapa (11 de 20 participantes), lo que representa 5.07\% de los entrevistados en el experimento.

En relación con los cambios de preferencia entre los participantes, el análisis de la matriz de transición presenta información de dichas conductas, por ejemplo, los nanoemprendedores que en la primera etapa prefieren seguir en la informalidad o ser formales en la segunda. Para explicar lo anterior, hay que tener en claro que este tipo de información se encuentra en las celdas que no están en la diagonal principal.

De acuerdo con lo señalado, en la celda $i=1$ y $j=2$ se observa la cantidad de participantes que en la primera etapa mostraban una preferencia por seguir en la informalidad, pero que en 
la segunda eligieron ser formales. Este porcentaje es de $32.65 \%$ y representa $22.12 \%$ del total de entrevistados en el experimento. También se aprecia que la transición de participantes en la categoría de informal hacia la incertidumbre o indecisión fue de $5.45 \%$ (celda $i=1$ y $j=$ 3), la cual representa un 3.69\% del total de nanoemprendedores en el experimento.

En lo que respecta a la categoría de los participantes indecisos, se nota que ninguno $(0.0 \%)$ en la primera etapa prefiere pasar a la informalidad en la segunda (celda $i=3, j=1$ ), mientras que $45 \%$ (celda $i=3, j=2$ ) cambió hacia la formalidad. En relación con la submuestra de 217 participantes, estos porcentajes fueron de $0.0 \%$ y $4.15 \%$, respectivamente.

\section{Estimaciones del modelo logit}

La Tabla 3 presenta los resultados de la estimación de tres modelos logit con el fin de examinar el impacto de diferentes factores que motivan el cambio de preferencias en los nanoemprendedores (primer modelo). El segundo modelo estima el impacto de las variables independientes para la preferencia por la informalidad y el tercero para la formalidad. Recordemos que en cada modelo la variable dependiente es binaria $(1=$ si se prefiere a la categoría en cuestión, y de 0 si no). Para cada modelo se tienen como variables independientes los cuatro grupos definidos en la sección previa (aspectos socioeconómicos, del entorno, simbólicos y preferencias).

\section{Tabla 3}

Efectos marginales de las variables independientes para los modelos logit

\begin{tabular}{lccc}
\hline \multirow{1}{*}{ Variables } & & \multicolumn{1}{c}{$d y / d x$} & \\
Edad & Total & Informalidad & Formalidad \\
& -.001 & .004 & -.005 \\
\hline \multirow{nyyy}{*}{ Casado } & $(.002)$ & $(.008)$ & $(.009)$ \\
\multirow{2}{*}{ Hombre } & $.069^{* *}$ & $-.134^{* *}$ & $.117^{* *}$ \\
& $(.032)$ & $(.059)$ & $(.049)$ \\
Escolaridad & $.095^{* *}$ & $.094^{* *}$ & $-.091^{* *}$ \\
& $(.044)$ & $(.043)$ & $(.043)$ \\
Clase & .013 & -.016 & .004 \\
& $(.043)$ & $(.060)$ & $(.033)$ \\
Inversión & .016 & $.027^{*}$ & .014 \\
& $(.013)$ & $(.016)$ & $(.017)$ \\
& .000002 & -.00002 & .00001 \\
& $(.00001)$ & $(.00002)$ & $(.00001)$
\end{tabular}




\begin{tabular}{|c|c|c|c|}
\hline \multirow{2}{*}{ Variables } & \multicolumn{3}{|c|}{$d y / d x$} \\
\hline & Total & Informalidad & Formalidad \\
\hline \multirow[t]{2}{*}{ Ganancias } & .000006 & $.00002^{* *}$ & $-.000018^{*}$ \\
\hline & $(.00001)$ & $(.00001)$ & $(.00001)$ \\
\hline \multirow[t]{2}{*}{ Promesas } & -.043 & .053 & .038 \\
\hline & $(.039)$ & $(.061)$ & $(.051)$ \\
\hline \multirow[t]{2}{*}{ Inseguridad } & .011 & $.012^{*}$ & .015 \\
\hline & $(.059)$ & $(.007)$ & $(.048)$ \\
\hline \multirow[t]{2}{*}{ Desempleo } & $.111^{* * *}$ & $.122^{* *}$ & .106 \\
\hline & $(.038)$ & $(.052)$ & $(.071)$ \\
\hline \multirow[t]{2}{*}{ Mal pagado } & $.149^{* *}$ & $.109^{* * *}$ & $-.136^{* *}$ \\
\hline & $(.060)$ & $(.038)$ & $(.059)$ \\
\hline \multirow[t]{2}{*}{ Empresarial } & .046 & .021 & .022 \\
\hline & $(.181)$ & $(.179)$ & $(.144)$ \\
\hline \multirow[t]{2}{*}{ Mejorar ingreso } & $.100^{* *}$ & .087 & .070 \\
\hline & $(.051)$ & $(.172)$ & $(.133)$ \\
\hline \multirow[t]{2}{*}{ Oportunidad } & .048 & .003 & .001 \\
\hline & $(.191)$ & $(.181)$ & $(.149)$ \\
\hline \multirow[t]{2}{*}{ Única forma } & $.313^{*}$ & $.350^{* *}$ & -.227 \\
\hline & $(.167)$ & $(.161)$ & $(.152)$ \\
\hline \multirow[t]{2}{*}{ Informalidad } & $.311^{*}$ & $.182^{* *}$ & $-.138^{*}$ \\
\hline & $(.167)$ & $(.084)$ & $(.081)$ \\
\hline \multirow[t]{2}{*}{ Formalidad } & $.081^{* * *}$ & $-.069^{* *}$ & $.032^{* *}$ \\
\hline & $(.021)$ & $(.032)$ & $(.016)$ \\
\hline \multirow[t]{2}{*}{ Indeciso } & -.182 & -.191 & $.173^{* *}$ \\
\hline & $(.156)$ & $(.164)$ & $(.087)$ \\
\hline McFadden's R ${ }^{2}$ & 0.365 & 0.220 & 0.146 \\
\hline Correctly classified & $88.3 \%$ & $76.6 \%$ & $73.1 \%$ \\
\hline $\mathrm{Y}=\operatorname{Pr}(\mathrm{y})$ (predict) & 0.690 & 0.620 & 0.556 \\
\hline
\end{tabular}

* Significativo al 10\%. ** Significativo al 5\%.*** Significativo al 1\%. (Error estándar entre paréntesis) Fuente: Elaboración propia. 
En relación con las características socioeconómicas de los nanoemprendedores se aprecia que el coeficiente estimado para la variable Hombre es positivo y significativo en la categoría de informalidad, lo cual significa una preferencia de los participantes varones hacia la informalidad. En el mismo sentido, se puede concluir que estos participantes (hombres) sienten menor afinidad hacia la formalidad.

En cuanto a la variable Casado se aprecia que existe una mayor disposición a preferir la formalidad y una menor disposición a la informalidad. De igual manera, si se observa la variable Ganancias se puede destacar que la probabilidad de que los participantes elijan la informalidad debido a las ganancias que obtienen en este sector es mucho mayor que la de aquellos participantes que prefieren la formalidad.

Por otro lado, el coeficiente de la variable Clase es significativo únicamente para el modelo de la informalidad. Dicho valor positivo implica que a medida que el nanoemprendedor se encuentre en estratos sociales inferiores, hay mayor probabilidad de que elija la informalidad. Otro hallazgo interesante es el de aquellos participantes que en el experimento argumentaron que se encuentran en la informalidad a pesar de que exista inseguridad en su zona o lugar de trabajo, ya que se esperaría que dichos nanoemprendedores decidieran dejar la informalidad debido a las extorsiones, cobros de piso, robos, etcétera. Sin embargo, los resultados muestran un impacto positivo y significativo en la variable de Inseguridad con el modelo de la informalidad.

Respecto a las variables Desempleo, Mal pagado y Única forma, se identifica que tienen un impacto significativo en los tres modelos; por ejemplo, los tres factores mencionados son determinantes para que los participantes prefieran elegir la informalidad. Resulta llamativo el impacto negativo de la variable Mal pagado en el modelo de formalidad, ya que puede interpretarse que este efecto se reparte en la informalidad, pero con efecto positivo. Dicho de otra manera, los nanoemprendedores que se encuentran en la formalidad, pero por razones externas su utilidad no es redituable -como ellos quisieran... además, de que pagan impuestos y formas legales...- pueden preferir en algún momento pasar a la informalidad (i.e., se observa el coeficiente -0.012 que afecta negativamente a los participantes que señalan ser formales y beneficia, en mayor medida, a los informales, 0.016).

Por último, aunque no menos importante, para las variables de preferencia (Informalidad, Formalidad e Indeciso) se encuentran resultados interesantes; por ejemplo, en el modelo de la preferencia hacia la formalidad, si el participante seleccionaba por la informalidad en la primera etapa, el impacto era significativo y negativo. Al mismo tiempo, al analizar el valor del coeficiente para la variable Formalidad en el modelo de preferencia a favor de la informalidad, se observa que los nanoempresarios que preferían la formalidad en la primera etapa eran renuentes a elegir por la informalidad.

Un dato adicional que arrojan las estimaciones de las variables Informalidad, Formalidad 
e Indeciso es la seguridad de los participantes en cada categoría; por ejemplo, se aprecia que los nanoemprendedores que muestran "mayor seguridad en su decisión” son los que prefieren seguir en la informalidad (0.410), seguidos de los que prefieren la formalidad (0.554). Finalmente, el impacto de la variable Indeciso sólo es significativo para el modelo de formalidad, ya que los participantes que no tenían definida (o no quisieron contestar) su preferencia en la primera etapa mostraba una incidencia positiva y significativa sobre la probabilidad (0.096) de preferir por esta categoría en la segunda etapa.

\section{Discusión de resultados}

De acuerdo con los resultados de este estudio, se puede concluir que el experimento de laboratorio propuesto para identificar los factores condicionantes que influyen en la creación o preferencia de una nanoempresa coincide con los hallazgos que presentan los estudios de González (2015) y Valencia (2012) respecto a que la integración o formación de esta unidad económica, normalmente se da por iniciativa de una persona de tal forma que dicha relación se convierte en hombre-empresa. Esta relación realiza todas las funciones de una organización lucrativa, pero tiene entre sus características particulares el no estar dentro de la formalidad y el que quizás permanezca en esa condición durante mucho tiempo.

Es importante señalar que este estudio es pionero en utilizar un análisis de experimento controlado en el tema de nanoempresas y que además fue modelado con una matriz de transición y un modelo logit, ya que su principal propósito fue cuantificar los factores que motivaron el cambio de preferencias y creación de esta unidad económica dadas sus condiciones de informalidad, y observar si estos factores afectan en su transición hacia la formalidad. En este sentido, no fue posible encontrar estudios con resultados de la misma magnitud; de igual forma, no se encontraron estudios acerca de la aplicación de estas técnicas dándole un enfoque particular a las nanoempresas. Algunas investigaciones de corte internacional como las de Ljungberg (2011), García, et al. (2008), López, Lejarriaga e Iturrioz (2007), García y Fernández (2005), Lejarriaga (2005) y Lejarriaga (2003) abordan la temática del origen, creación y funcionamiento de las nanoempresas encontrando que dichas unidades económicas pueden formarse con una o hasta tres personas que tengan intereses en común para participar en cualquier mercado y, de esta manera, obtener ganancias lucrativas por los servicios, bienes o productos que ofrezcan. Mientras que estudios de corte nacional como los de Castillo y Vela (2013) y Aguilar, Mungaray y Ramírez (2014) encuentran que la influencia del entorno social y doméstico impacta en la elección del trabajo autónomo o por cuenta propia en México. Concretamente, se conjetura que junto a los aspectos estructurales de la economía que han impedido la creación de puestos de trabajo formales, existen algunos elementos socioculturales que promueven dicha elección, los cuales pueden relacionarse con las generaciones de los trabajadores, dado que los 
padres ocupados suelen transmitir cierto capital humano informal a sus descendientes, específicamente en las mujeres. De igual manera, Alvarado, Dávila y Vázquez (2018) y Alvarado, Ortiz y Morales (2018) establecen que las dimensiones o factores que pueden dar origen a la iniciación de empresas unipersonales son el desempleo, relaciones entre grupos o individuos afines, procesos demográficos, relaciones sociales (oportunidad de negocios), desinterés en procesos legales, relaciones de conflictos y horarios flexibles, así como también la intención de mejorar el ingreso familiar.

\section{Conclusiones}

En este artículo se llevó a cabo un experimento de laboratorio controlado de octubre de 2017 a marzo de 2018 en la ciudad de Monterrey en Nuevo León. En este lapso de tiempo se quiso conocer los factores que motivan la creación de nanoempresas. Además, se buscó cuantificar las diferencias entre los nanoemprendedores que se encuentran en la informalidad vs formalidad. La discusión derivada de este estudio permite confirmar la heterogeneidad de las personas participantes en el experimento, ya que al momento de crear sus nanoempresas los principales motivos que influyen son el desempleo, empleos mal pagados, mejorar el ingreso familiar y las ganancias que se obtienen por ser el único dueño o jefe de su empresa unipersonal. También se identificó que los participantes que se encuentran en la informalidad pueden cambiar de preferencia o transitar hacia la formalidad, siempre y cuando conozcan el proceso, trámites, requisitos y ventajas de tener una nanoempresa en el marco de la ley.

Los hallazgos de este trabajo nos permiten someter a consideración algunas acciones de política pública para evitar que los -futuros- nanoemprendedores o nanoempresarios elijan la informalidad. Desde políticas inmediatas o de corto plazo, como la mejora en los servicios de apertura de negocios, específicamente en los costos y tiempo de los trámites necesarios para obtener la licencia de funcionamiento, así como la creación de un departamento o área encargada de aplicar un modelo de intervención directa con todos los interesados en crear una nanoempresa que conlleve desde la gestión hasta la consolidación de la unidad económica, hasta políticas de largo plazo como implementar, a través del sistema educativo, la cultura de la legalidad, así como ferias o programas de emprendimiento con responsabilidad social.

Una condición para concretar lo anterior es detectar las zonas con mayor participación de informalidad, con la finalidad de brindar asesoría y capacitación a los nanoempresarios que conlleve diagnósticos, formación, acompañamiento, monitoreos, evaluaciones y culminación de nanoempresas formales y, de esta manera, minimizar las preferencias por seguir en la ilegalidad, ya que este sector genera una derrama económica significativa. No obstante, si los nanoempresarios transitan hacia la formalidad, sin duda alguna se generarían mayores externalidades positivas. 


\section{Limitaciones académicas}

Desde el punto de vista académico, el presente trabajo ha pretendido contribuir a los estudios sobre las nanoempresas, un tema que muchas veces ha sido relegado. La investigación se realiza en Monterrey, una de las ciudades más importantes de México. A nivel nacional, existen pocos trabajos empíricos sobre la cuantificación de aquellos factores que influyen en la creación de una nanoempresa, así como la aplicación de un experimento de laboratorio que permite identificar el cambio de preferencias de los nanoempresarios hacia la formalidad, ya que la mayoría de los estudios se enfoca en el análisis cualitativo y descriptivo sobre la informalidad. En nuestro caso, se emplea un modelo de elección discreta binaria tipo logit y se exploran los efectos o cambios de preferencia de las personas participantes a través de una matriz de transición.

A pesar de que el análisis realizado en esta investigación pueda tener limitaciones en lo concerniente a la representatividad de la muestra o sesgos en las técnicas utilizadas, entendemos que ello conforma un procedimiento válido para representar la variación sistemática de un conjunto de datos de corte longitudinal. De la misma manera, se ha intentado desarrollar un trabajo riguroso en lo que respecta a la aplicación de un experimento de laboratorio controlado en las ciencias sociales. En este sentido, somos conscientes de las limitaciones y de las oportunidades de mejora; por ejemplo, a nivel conceptual, los resultados han planteado algunas cuestiones que exigen el estudio de otras variables que no han sido analizadas en este trabajo y que podrían mejorar el conocimiento sobre la probabilidad de que los nanoempresarios transiten hacia la formalidad, tales como realizar un análisis comparativo por municipios, regiones, entidades federativas, género y nivel educativo.

\section{Referencias}

Acs, Z. (2006). How is entrepreneurship good for economic growth? Innovations Technology Governance Globalization, 1(1), 97-107. DOI: https://doi.org/10.1162/itgg.2006.1.1.97

Aguilar, J.G., Mungaray, A.B. y M. Ramírez. (2014). Género y autoempleo en México durante la recesión de 2008-2009: un estudio de flujos laborales. Papeles de Población, 80, 145-191.

Alonso, M. y Galve, C. (2008). El emprendedor y la empresa: una revisión teórica de los determinantes a su constitución. Acciones e Investigaciones Sociales, 26, 5-44. DOI: https://doi.org/10.26754/ ojs_ais/ais.200826332

Alvarado, E., Dávila, M.C. y M.A. Vázquez. (2018). Factors that influence the continuity and survival of a micro-business in Mexico. Contaduría y Administración, 63(3), 1-20. DOI: http://dx.doi. org/10.22201/fca.24488410e.2018.1281

Alvarado, E., Ortiz, J. y D. Morales. (2018). Factores que influyen en el emprendimiento de un micronegocio familiar en México. Revista de Economía del Rosario, 21(1), 153-180. http://dx.doi. org/10.12804/revistas.urosario.edu.co/economia/a.6816 
Álvarez, A. y Valencia, P. (2008). Un análisis de los factores sociodemográficos determinantes en la creación de empresas en el marco de la interculturalidad. Revista de Economía Mundial, 18, 341353.

Anthopoulou, T. (2010). Rural women in local agrofood production: Between entrepreneurial initiatives and family strategies. A case study in Greece. Journal of Rural Studies, 26(4), 394-403. DOI: https://doi.org/10.1016/j.jrurstud.2010.03.004

Audretsch, D. y Fritsch, M. (1994). The geography of firm births in Germany. Regional Studies, 28(4), 359-365. DOI: https://doi.org/10.1080/00343409412331348326

Backes, U. y Moog, P. (2013). The disposition to become an entrepreneur and the jacks-of-all-trades in social and human capital. The Journal of Socio-Economics, 47, 55-72. DOI: https://doi. org/10.1016/j.socec.2013.08.008

Baptista, R., Karaöz, M. y J. Mendonça. (2014). The impact of human capital on the early success of necessity versus opportunity-based entrepreneurs. Small Business Economics, 42(4), 831-847. DOI: I 10.1007/s11187-013-9502-z

BM-Banco Mundial. (2004). La pobreza en México. Una evaluación de las condiciones, las tendencias y la estrategia del gobierno (1ra. ed.). Washington, D.C.: Banco Internacional de Reconstrucción y Fomento / Banco Mundial.

Budnick, F. (2007). Matemáticas aplicadas para administración, economía y ciencias sociales (4ta. ed.). México: McGraw-Hill.

Casas, E. e Ibarra, L. (2013). Análisis del impacto de crecimiento en las Pymes que se desarrollaron bajo un esquema de incubación: caso de estudio. Revista Internacional Administración y Finanzas, 6(3), 85-94.

Castillo, D. y Vela, F. (2013). Movilidad laboral y trasmisión intergeneracional del autoempleo informal en México. Revista Gaceta Laboral, 19(1), 5-35.

Chávez, M. (2019). El valor de la economía informal. Contenido, 1-4. Disponible en: https://contenido.com.mx/2019/06/el-valor-de-la-economia-informal/

Coleman, J.S. (1988). Social capital in the creation of human capital. The American Journal of Sociology, 94, 95-120. DOI: https://www.jstor.org/stable/2780243

COPARMEX-Confederación Patronal de la República Mexicana. (2019). Mínimo crecimiento del empleo formal. Comunicado COPARMEX, 1-2. Disponible en: https://coparmex.org.mx/minimo-crecimiento-del-empleo-formal-coparmex/

Davidsson, P. y Honig, B. (2003). The role of social and human capital among nascent entrepreneurs. Journal of Business Venturing, 18(3), 301-331. DOI: https://doi.org/10.1016/S08839026(02)00097-6

De la Garma, F. (2010). La economía informal en México: una primera aproximación. Tesis de Maestría. Escuela de Graduados en Administración Pública y Política Pública, Instituto Tecnológico de Estudios Superiores de Monterrey.

Delgado, S.E., Cruz, L. y E. Lince. (2019). Modelo de gestión de análisis administrativo y contable para nano y MIPYMES. En Cruz, et al. (Coords.). Innovación en Ingeniería Financiera (pp. 150160), Xalapa, Veracruz, México: Editorial Red Iberoamericana de Academias de Investigación.

Elston, J. y Audretsch, D. (2011). Financing the entrepreneurial decision: an empirical approach using experimental data on risk attitudes. Small Business Economics, 36(2), 209-222. DOI: https://doi. org/10.1007/s11187-009-9210-x

Fuentes, N., G. Osorio y A. Mungaray. (2016). Capacidades intangibles para la competitividad microempresarial en México. Revista Problemas del Desarrollo, 186(47), 83-106.

Fundación $1^{\circ}$ de Mayo. (2011). Reflexiones y propuestas en torno a la economía social y el autoempleo (1ra. ed.). Madrid, España: Ministerio de Trabajo e Inmigración y de la Fundación Formación y Empleo (FOREM). 
García, C. y Fernández, J. (2005). El empresario individual: situación actual y propuestas de actuación futuras. Revista de Economía Pública, Social y Cooperativa, 52, 201-217.

García, C., Lejariaga, G., Bel, P., Fernández, J. y S. Martín. (2008). El emprendimiento y el empleo a través de la empresa individual: Contrastes de fuentes estadísticas. Revista de Estudios Cooperativos, 96, 16-48.

García, C., Martínez, A. y R. Fernández. (2010). Características del emprendedor influyentes en el proceso de creación empresarial y en el éxito esperado. Revista Europea de Dirección y Economía de la Empresa, 19(2), 31-48.

Garza, H.A. (2010). Empresas unipersonales en México. Tesis de Maestría. Escuela de Graduados en Administración Pública y Política Pública, Instituto Tecnológico de Estudios Superiores de Monterrey.

Gelderen, M., Bosma, N. y R. Thurik. (2001). Setting up a business in the Netherland: Who starts, who gives up, who is still trying. Programme Research SMEs \& Entrepreneurship. Disponible en: https://www.semanticscholar.org/paper/Setting-Up-a-Business-in-the-Netherlands\%3A-WhoWho-Gelderen-Thurik/ad00e1612d3ba9430375555185a43c1498a13d79\#citing-papers

Gibb, A. y J. Ritchie. (1982). Understanding the process of starting small businesses. European Small Business Journal, 1(1), 26-47. DOI: https://doi.org/10.1177/026624268200100102

González, A. (2015). La nanoempresa, como una forma de organización económica, su reconocimiento para México. Revista Venezolana de Análisis de Coyuntura, 21(1), 175-186.

Kantis, H. (2003). Componentes macroeconómicos, sectoriales y microeconómicos para la estrategia nacional de desarrollo. Lineamientos para fortalecer las fuentes de crecimiento económico. Programa Multisectorial de Preinversión II préstamo BID 925 OC-AR, Estudio 1.EG.33.3, estudios sobre empleo, componente en: Creación y fortalecimiento de nuevas empresas. Buenos Aires, Argentina: Ministerio de Economía de la Nación, Secretaría de Política Económica, Unidad de Preinversión (UNPRE).

Kantis, H., Ishida, M. y M. Komori. (2002). Empresarialidad en economías emergentes: Creación y desarrollo de nuevas empresas en América Latina y el Este de Asia (1ra. ed.). Banco Interamericano de Desarrollo.

Keeble, D. y Walker, S. (1994). New firms, small firms and dead firm's spatial patterns and determinants in the United Kingdom. Regional Studies, 28(4), 411-427. DOI: https://doi.org/10.1080/003 43409412331348366

Kim, P.H., Aldrich, H.E. y L.A. Keister. (2006). Access (not) denied: the impact of financial, human, and cultural capital on entrepreneurial entry in the United States. Small Business Economics, 27, 5-22. DOI: https://doi.org/10.1007/s11187-006-0007-x

Kolman, B. y Hill, D. (2006). Álgebra lineal (8va. ed.). México: Pearson Prentice-Hall.

Lejarriaga, G. (2003). La sociedad laboral como nanoempresa: hacia la sociedad laboral unipersonal. Revista de las Sociedades Laborales Madrileñas, 5, 1-24.

Lejarriaga, G. (2011). Ni todas las organizaciones son empresas, ni todas las empresas son organizaciones. El caso de las nanoempresas (pp. 57-75). En E. Ibarra, T. Nilda y A. Poggiese (Coords.). III Jornada de Administración del NEA y I Encuentro Internacional de Administración. Argentina: Universidad Nacional de Misiones.

Lejarriaga, G., Durán, B. y M. Miranda. (2005). La sociedad laboral y su capacidad de creación de empleo. Una aproximación con base en la sociedad laboral de la Ciudad de Madrid. Revista de Estudios Cooperativos, 87, 107-138.

López, S., Lejarriaga, G. y J. Iturrioz. (2007). Consideraciones sobre la naturaleza del capital social en las sociedades cooperativas de trabajo asociado. Revista de Estudios Cooperativos, 91, 93-119.

Martínez, E. (2008). Logit Model como modelo de elección discreta: origen y evolución. Anuario Jurídico y Económico Escurialense, XLI, 470-483. 
Ministerio de Economía. (2002). Encuentro de Pequeñas y Medianas Empresas. "Hacia un nuevo entorno para el emprendedor del Siglo XXI". Documento de Trabajo del Grupo "Los empresarios autónomos". Móstoles, Madrid, España: Universidad Rey Juan Carlos.

Morales, J., Bustamante, A., Vargas, S., Pérez, N. y O. Sereno. (2015). Factores de éxito emprendedor en dos municipios de la montaña de Guerrero, México. Nova Scientia, 7(3), 416-435.

Narayan, D. y Pritchett,L. (1999). Cents and Sociability; household income and social capital in Rural Tanzania. Economic Development and Cultural Change, 47(4), 871-897. DOI: 10.1086/452436

Nava, E. (2013). Factores que Influyen la Creación de Empresas Pymes y Empresas Familiares. International Journal of Good Conscience, 8(1), 11-22.

Observatorio Europeo de las PYME. (2018). Annual Report on European SMEs 2017/2018 (10a. ed.). Observatorio Europeo / European Union, Luxembourg. DOI: 10.2873/248745.

OIT-Organización Internacional del Trabajo. (2014). El empleo informal en México: situación actual, políticas y desafíos. Programa de Promoción de la Formalización en América Latina y el Caribe. Disponible en: https://www.ilo.org/wcmsp5/groups/public/---americas/---ro-lima/documents/publication/wcms_245619.pdf

Palacio, L.A. y Parra, D.F. (2012). Economía experimental: un panorama general. Revista Lebret, 4, 277-294. DOI: https://doi.org/10.15332/rl.v4i4.338

Pazmiño, W.E., Merchán, V.A. y E.H. Jiménez. (2018). Comercio informal y su incidencia en los habitantes de la Ciudad de Babahoyo, Provincia de los Ríos, Ecuador. Revista Dilemas Contemporáneos: Educación, Política y Valores, 6(56), 1-22.

Pérez, C. (2005). Métodos estadísticos avanzados con SPSS (1ra. ed.). Madrid: Editorial Thomson.

Pinillos, M. (2001). Factores coadyuvantes para la creación de empresas en la Comunidad de Madrid. Papeles de Economía Española, 89(90), 343-361.

Quintana, C. (2001). Dimensiones del éxito de las empresas emprendedoras. Investigaciones Europeas de Dirección y Economía de la Empresa, 7(2), 139-158.

Raydán, S. (2010). Guía para no meter la pata. Consejos empresariales para el nano emprendedor. Caracas, Venezuela: FUNDEFIR-Dirección de Desarrollo Sostenible Total.

Robles, D. y Martínez, M.A. (2018). Determinantes principales de la informalidad: un análisis regional para México. Región y Sociedad, 30(71), 1-35. DOI: https://doi.org/10.22198/rys.2018.71.a575

Rodríguez, A. (2006). El emprendedor venezolano. Debates IESA, 11(3), 20-22.

Rodríguez, A. y Palavicini, E.I. (2013). Does local economic development really work? Assesing LED across Mexican municipalities. Geoforum, 44, 303-315. DOI: https://doi.org/10.1016/j.geoforum.2012.07.010

Scott, J. (2009). Gender and entrepreneurship: A multilevel theory and analysis. International Journal of Entrepreneurial Behavior \& Research, 15(4), 386-388. DOI: https://doi. org/10.1108/13552550910967958

Sigalia, R.J. y Carney, S. (2012). Poverty reduction through entrepreneurship: microcredit, learning and ambivalence amongst women in urban Tanzania. International Journal of Educational Development, 32, 546-554. DOI: https://doi.org/10.1016/j.ijedudev.2012.02.011

Texis, M., Ramírez, M. y J.G. Aguilar. (2016). Microempresas de base social y sus posibilidades de supervivencia. Contaduría y Administración, 61(3), 51-567. DOI: https://doi.org/10.1016/j. cya.2015.04.001

Valdés, A. (2004). Tipología del trabajo autónomo. Revista de Relaciones Laborales, Economía, Sociología del Trabajo y Trabajo Autónomo, 70(1), 11-58.

Valencia, G.D. (2012). Autoempleo y emprendimiento. Una hipótesis de trabajo para explicar una de las estrategias adoptadas por los gobiernos para hacer frente al progreso del mercado. Semestre Económico, 15(32), 103-128.

Walton, M. y López, G. (2005). La pobreza en México. Una evaluación de las condiciones, las tendencias y la estrategia del gobierno. Informes del Banco Mundial, 61, 1-4. 